Quand la voix devient la lettre : les anciens manuscrits autochtones (puta tupuna) de Polynésie française

\title{
Bruno Saura
}

\section{(2) OpenEdition Journals}

Édition électronique

URL : http://journals.openedition.org/jso/2592

DOI : $10.4000 /$ jso. 2592

ISSN : $1760-7256$

Éditeur

Société des océanistes

Édition imprimée

Date de publication : 15 décembre 2008

Pagination : 293-310

ISBN : 978-2-85430-012-3

ISSN : 0300-953x

Référence électronique

Bruno Saura, "Quand la voix devient la lettre : les anciens manuscrits autochtones (puta tupuna) de Polynésie française », Journal de la Société des Océanistes [En ligne], 126-127 | Année 2008, mis en ligne le 15 décembre 2011, consulté le 16 juin 2020. URL : http://journals.openedition.org/jso/2592 ; DOI : https://doi.org/10.4000/jso.2592 


\section{Quand la voix devient la lettre : les anciens manuscrits autochtones (puta tupuna) de Polynésie française}

par

Bruno SAURA *

\section{RÉSUMÉ}

Les manuscrits écrits par des autochtones sont apparus au XIX ${ }^{e}$ siècle en Polynésie française dans des conditions précisées ici, avec un développement varié selon les archipels. Sont présentés surtout ceux des îles Australes, particulièrement nombreux. Puis sont étudiés deux textes publiés récemment, relatifs aux îles Sous-le-Vent, dont un récit sur la famille royale des Tamatoal de Ra'iātea. Ils livrent une histoire intérieure, spécialement intéressante à analyser. Ces manuscrits traditionnels dits puta tupuna ne doivent pas être confondus avec des textes plus récents, qui s'en inspirent mais sont nés de la rencontre avec des æuvres occidentales modernes.

MoTS-CLÉS : manuscrits polynésiens, histoire autochtone, écriture, littérature, traditions, généalogies

Le titre du présent article est un hommage au célèbre ouvrage de Paul Zumthor La lettre et la voix (1987) consacré aux ouvres vocales du Moyen Âge occidental. Paul Zumthor y démontre combien le fait d'aborder la « littérature " médiévale uniquement sous une forme textuelle (celle par laquelle nous la connaissons aujourd'hui) ampute cette « littérature » de ce qui la caractérise : l'oralité, doublée

\begin{abstract}
The first native manuscripts appeared in French Polynesia in the XIX ${ }^{\text {th }}$ century, in a context hereafter precised, with various developments according to the different groups of islands. This article includes a presentation of writings from the Austral islands, which are significantly numerous. Then are introduced two manuscripts recently published in Tahiti, about the Leeward islands, one of them dealing with the history of the Tamatoa royal family from Ra'iätea. They both constitute a valuable interior Polynesian history and literature. These traditional writings, called puta tupuna, must be distinguished from more recent works, based on them but influenced by contemporary western writings.
\end{abstract}

KeYwords: manuscripts, local history, Polynesian writings, literature, traditions, genealogies

de la théâtralité. En Occident, l'invention du roman - à partir du XII siècle -, puis celle des techniques d'imprimerie engagent le passage d'une culture principalement orale vers une culture dans laquelle les récits circulent plus massivement et sont reçus par d'autres que leurs destinataires originaux. La voix devient la lettre, mais d'emblée, la lettre est autre chose que la voix ${ }^{2}$.

* Professeur, université de la Polynésie française, bruno.saura@upf.pf 
Très loin des terres d'Europe, nous nous intéresserons ici à un processus similaire par lequel la voix se fit et continue de devenir la lettre. Il offre le grand intérêt d'être observable dans un temps très proche de nous : du XIX ${ }^{\mathrm{e}}$ siècle à aujourd'hui. Il concerne une société jusque-là exclusivement de tradition orale : les îles de la Société (îles Du-Vent et Sous-le-Vent) et les îles Australes, dans l'actuelle Polynésie française. Dans cet ensemble, l'introduction de l'écriture, dans le sillon des conversions au christianisme des années 1820, a produit l'apparition de manuscrits autochtones, dits puta tupuna, dont certains sont désormais partiellement ou intégralement publiés, tandis que d'autres continuent à être recopiés, voire rédigés.

Nous présenterons les principaux de ces textes, en commençant par quelques réflexions générales sur leurs conditions d'écriture (lieu, période, auteurs...). Puis, sur la base de cahiers de traditions des îles Sous-le-Vent, nous montrerons leur hétérogénéité ainsi que leur intérêt en matière d'étude d'une histoire polynésienne intérieure et du rapport des Polynésiens à leur passé. Suivra l'étude de textes plus récents, notamment ceux de l'auteur tahitien Charles Manutahi qui, s'ils se veulent basés sur des cahiers de traditions voire s'ils sont présentés comme tels, en constituent en fait une réécriture contemporaine ésotérique.

\section{Caractéristiques générales de ces textes}

Si Alain Babadzan dans un article fondateur (1979) date des années 1885-1890 l'apparition des puta tupuna à Rurutu, le plus ancien puta tupuna que nous ayons pu identifier en Polynésie française a été rédigé en 1846, aux îles Sous-leVent. Il a été publié en l'an 2000 (avec une édition critique) par le ministère de la Culture de la Polynésie française, sous le titre Histoire et traditions de Huahine et Porapora (Borabora). La plupart des autres puta tupuna connus ont plutôt été rédigés vers la fin du XIX ${ }^{\mathrm{e}}$ siècle; ils proviennent des Australes et des îles Sous-le-Vent, plus rarement des îles Du-Vent (Tahiti et Mo'orea) ou des Tuamotu-Gambier. Nous n'en connaissons aucun des îles Marquises.

Ces anciens manuscrits autochtones ont généralement la forme de cahiers grand format à la couverture cartonnée, du type registre. Ils sont apparus avec la circulation de tels supports à Tahiti et dans les îles voisines, principalement dans la deuxième moitié du XIx ${ }^{\mathrm{e}}$ siècle, même si l'apprentissage de l'écriture par les habitants de certaines îles avait commencé dans la première moitié du XIX ${ }^{\mathrm{e}}$ (Parsonson, 1967). Ils sont rédigés à l'encre, la plupart du temps d'une écriture régulière, la qualité graphique de l'écriture s'accroissant souvent au fil des premiers recopiages dont ces textes ont fait l'objet. En raison de la dégradation du papier dans les îles tropicales, les cahiers parvenus jusqu'à notre époque ont la plupart du temps été recopiés une ou plusieurs fois, le recopiage pouvant altérer le texte original de diverses manières : erreurs dans le recopiage proprement dit, suppressions volontaires, ajouts d'interprétations ou de développements à l'intérieur du texte, ajout de textes à la suite de celui-ci.

En début ou en fin de document figurent souvent le nom de l'auteur et la date de rédaction finale du texte original. L'auteur (ou les auteurs) des recopiages successifs précisent parfois sa (ou leur) propre identité ainsi que la date ou période $\mathrm{du}$ recopiage. L'absence de ces indications fait obstacle à une appréciation exacte du texte dans le sens où elle peut conduire les lecteurs d'une copie à attribuer au premier auteur d'un manuscrit certaines informations en fait apparues au fil de son recopiage. Ces manuscrits sont ordinairement désignés dans l'ensemble tahitien sous l'appellation puta (de l'anglais book) suivie d'un qualificatif : puta tupuna " livre [des] ancêtres », puta tumu « livre [des] origines », puta 'a'amu "livre [d']histoires », puta parau pa'ari « livre [de] paroles traditionnelles ». Ces appellations se recouvrent largement. Quel est leur contenu ? Ils portent différents types d'informations, de paroles $\left(\right.$ parau $\left.^{3}\right)$ devenues écriture (papa'i, papa'ira'a), à l'intérieur d'un éventail maximal pouvant comprendre :

- des généalogies ;

- des traditions relatives à des divinités, objets, lieux, héros ou personnages historiques préchrétiens ;

- des calendriers lunaires ;

- des recettes de pharmacopée traditionnelle ;

- des narrations d'actions de personnages de l'époque du contact avec les Européens, avec évocation des processus survenus à cette époque : acculturation, christianisation, parfois colonisation ;

- parfois des commentaires de versets bibliques, chants religieux chrétiens ou autres écrits liés à la vie paroissiale.

3. Autour de la notion de la tradition orale en Polynésie, voir notamment Flora Devatine (1979) et Vahi Tuheiava-Richaud (1999). 
Comme nous avons pu nous en rendre compte à Rurutu, il arrive que la copie d'un puta original s'enrichisse de nos jours d'une section comprenant des panneaux du code de la route ou une recette de gâteau de mariage... soit autant de données particulièrement importantes à un moment de la vie d'un membre de la famille. C'est dire qu'on aurait tort de doter les puta tupuna d'une sacralité qu'ils n'ont pas toujours ${ }^{4}$, même si bien souvent, la nature - religieuse - de certaines données qu'ils contiennent, ajoutée au respect de la mémoire de l'ancêtre à l'origine de sa rédaction ou de sa copie, leur confère une importance réelle. Qui plus est, ceux qui ne disposent pas de la copie d'un puta lui prêtent d'autant plus de prix qu'ils l'imaginent contenir des informations importantes en matière de généalogie et donc de droits fonciers, dont se serviraient ceux qui le détiennent. C'est ainsi qu'à l'intérieur même d'une famille, il n'est pas rare que l'un des parents soit soupçonné voire accusé ponctuellement de détenir un puta hérité d'un ancêtre commun, puta prétendument égaré mais en fait précieusement conservé.

Puta volés, puta confisqués, détournés... mais aussi puta enfouis, les «mythes » modernes ne manquent pas au sujet de ces écrits anciens ; l'un des plus tenaces a trait à ces puta qui auraient été ensevelis avec leur propriétaire, à la mort de celui-ci, suivant sa volonté. La chose est bien attestée concernant l'orateur tahitien Pouira Teauna dit Tearapō (1902-1969), qui fut inhumé avec ses précieux cahiers de traditions... ${ }^{5}$ La disparition - avérée ou non - de puta tupuna, dans ces conditions, heurte nombre de Polynésiens d'aujourd'hui qui y voient un acte égoïste ${ }^{6}$ signifiant aux gens du temps présent qu'ils ne méritent pas de conserver des traditions dont, en fait, l'auteur ou l'héritier des cahiers ensevelis n'était pourtant lui-même que le détenteur momentané. Nous nous attarderons maintenant sur les puta tupuna de Rurutu, particulièrement nombreux.

\section{Les puta tupuna de Rurutu (îles Australes)}

Les Australes passent pour un berceau fécond en matière de puta tupuna ou, plutôt, pour avoir été le lieu d'écriture de nombre de puta tupuna connus et conservés, ce qui ne signifie pas nécessairement la même chose. Les deux processus coexistent en fait, à savoir, d'une part, l'apparition de multiples manuscrits dans ces îles (par rapport à d'autres) - pour des raisons que nous allons expliciter - et, d'autre part, la conservation particulière de certains de ces textes. Sur le premier point, le fait est que les Australes comme les îles de la Société sont de hauts lieux de la culture protestante polynésienne. Dans ces îles, nombre d'habitants, initiés depuis le XIX ${ }^{\mathrm{e}}$ siècle à la lecture de la Bible en tahitien, ont participé très tôt à la culture de l'écriture; de là, le fait que les textes que certains individus ont alors entrepris de rédiger, qu'ils soient relatifs aux temps pré-chrétiens ou à des sujets chrétiens, ont été rédigés en tahitien, même si presque chaque île des Australes possède une langue spécifique. Lisant la Bible en tahitien, célébrant le culte dans cette langue, certains habitants des Australes, lorsqu'ils se sont mis à écrire, l'ont fait en tahitien ou dans une mezza lingua proche $\mathrm{du}$ tahitien.

Souvent, les auteurs de ces manuscrits figurent parmi les meilleurs lettrés d'une île : dignitaires protestants, diacres ou pasteurs, ainsi que des chefs de district, des juges et grands juges, soit autant d'hommes de la loi ou de la Loi. Ils sont tous de sexe masculin, ce qui s'explique, en milieu protestant, par une meilleure familiarité des hommes avec l'écriture - favorisant l'accès aux fonctions de pasteur et de diacre-; aux XIX ${ }^{\mathrm{e}}$ et $\mathrm{Xx}^{\mathrm{e}}$ siècles, les chefs de district sont aussi généralement des hommes, mais pas exclusivement. À notre connaissance, aucun manuscrit ancien de Polynésie française n'a été rédigé par une femme ${ }^{7}$.

Pourquoi et pour qui avoir écrit ces textes? La question est d'autant plus légitime que nombre de sujets abordés ont trait aux temps pré-

4. Alain Badadzan remarque qu'à Rurutu, le contenu des puta tupuna est «assez disparate, à savoir que s'y trouvent juxtaposés (et apparemment mis sur le même plan) des textes de nature différente » (1979: 226). Au sujet du Moyen Âge, Zumthor relevait également «l'absence, avant le $\mathrm{XIV}^{\mathrm{e}}$ siècle, de toute idée de cohérence textuelle dans la composition des manuscrits... » $(1987: 122)$.

5. Claude Marere (journaliste puis responsable du service de presse de la présidence de la Polynésie française sous les gouvernements d'Oscar Temaru, 2004-2008) déplore ainsi "tous les "puta tupuna" qui pourrissent dans les tombes de cimetières, à Bora-Bora, à Arue, à Papara ou ailleurs » $(1982: 8)$.

6. Un mépris assez similaire préside à la rédaction de ses Mémoires (1972) par la reine Marau Taaroa. Il la conduit malgré tout à une démarche positive puisqu'elle condescend à transmettre par écrit son savoir relatif au Tahiti aristocratique des temps anciens.

7. Sur ce point, on doit à nouveau mentionner l'ouvrage Les mémoires de Marau Taaroa (1860-1934), dernière reine de Tahiti - car épouse de Pomare v. Si son but est aussi la conservation de traditions familiales polynésiennes, il ne s'agit pas d'un puta tupuna (manuscrit) privé, mais d'un ouvrage rédigé principalement en anglais (Marau Taaroa Salmon étant anglaise par son père). Sa structure est celle d'une œuvre historico-littéraire construite. Ce livre se distingue donc, à la fois formellement et par 
chrétiens, alors que leurs auteurs figurent souvent parmi les plus zélés convertis protestants autochtones. En fait, comme l'a justement souligné Alain Babadzan (1979 : 226) et comme le montrent aussi les puta publiés jusqu'ici dans la série des Cahiers du patrimoine (Saura et al., 2000 et 2003), même lorsqu'ils évoquent les temps pré-chrétiens, les auteurs de ces manuscrits le font en adoptant une perspective chrétienne de distanciation : les anciennes statues et objets de culte sont assez systématiquement présentés comme des « idoles » (néologisme itoro), etc. Toutefois, ces nouveaux lettrés ont souhaité transcrire tout ou partie de leurs traditions, preuve que l'acculturation chrétienne (processus volontaire, qui ne saurait être confondu avec la colonisation) a certes opacifié le regard porté sur les temps anciens, mais n'en a pas tout effacé. Les auteurs ont transcrit des généalogies servant à définir leur identité familiale et à asseoir des droits politiques et fonciers (Saura, 2008); la mémoire de mythes et de légendes préchrétiennes non liés à des territoires a été également transmise. Faisant écho aux personnages de l'Ancien Testament dont la fréquentation via le texte biblique leur est devenue désormais quotidienne (Saura, 2004), ces Polynésiens du XIX ${ }^{\mathrm{e}}$ siècle, tels les juges, prêtres et autres rois de l'Ancien Testament, ont intériorisé une sacralité de l'écriture, bien protestante - voir Daniel Mauer (1972) et Jacques Nicole (1988) -, qui les a poussés à leur tour à transmettre un témoignage de leurs origines, pour mémoire. Ils comprennent l'évocation des temps anciens (païens), suivie de celle des temps chrétiens - avec parfois l'aménagement d'une transition syncrétique entre les deux périodes et systèmes de représentations, très évidente à Rurutu (Babadzan, 1979 et 1982).

Si la question de la langue et de la religion des missionnaires à l'origine des conversions et de l'alphabétisation explique la fréquence d'apparition de puta aux Australes et îles Du-Vent, elle permet de comprendre, a contrario, la quasiabsence de tels cahiers d'écrits autochtones aux îles Marquises : là, les habitants ont principale- ment été évangélisés par des pères catholiques qui célébraient la messe en latin et étaient moins impliqués que les missionnaires protestants dans l'œuvre d'alphabétisation. Il n'existe ainsi pas à ce jour de traduction intégrale de la Bible en marquisien ; la seule disponible dans ces îles est, depuis 1838 (date de son impression), la traduction de la Bible (protestante) en tahitien, à laquelle s'ajoutent néanmoins quelques textes des évangiles traduits en marquisien au début du $\mathrm{xx}^{\mathrm{e}}$ siècle.

Malgré cette moindre culture du livre et de l'écriture, en milieu catholique que protestant, dans quelques îles catholiques, certains pères ont encouragé la rédaction de récits traditionnels en langue vernaculaire. C'est le cas de Mangareva (Gambier) où les pères Maigret, Laval et Liausu commencent par transcrire et publier dès les années 1840 des textes autochtones ${ }^{8}$ avant qu'un originaire de l'île, le père Tiripone Putairi (18461881) rédige dans les années 1870 un texte intitulé E atoga Magareva mei te ao eteni roa (Histoire de Mangareva depuis les temps du paganisme les plus reculés). Toutefois, ce texte ne relève pas d'une écriture personnelle; il est plutôt une adaptation de certains chapitres de l'ouvrage alors en cours de rédaction par le père Honoré Laval (publié en 1938 sous le titre Mangarareva. L'histoire ancienne d'un peuple polynésien. Mémoires ethnographiques...) sur la base de la tradition orale de l'île.

Rares sont les puta tupuna proprement dits relatifs à l'île de Tahiti. Cela pourrait s'expliquer par des raisons matérielles (la conservation de ces registres s'étant peut-être opérée moins bien lors des mouvements de population - plus importants ici qu'ailleurs -), mais aussi culturelles. Il est ainsi possible qu'à Tahiti, la diffusion de la langue française dans les milieux lettrés polynésiens (et notamment « demis »- ou métis-) ait pu freiner l'écriture de textes en tahitien, sans pour autant produire un nombre important de recueils de traditions en français.

Une autre explication de la relative rareté des puta de Tahiti et même des îles Sous-le-Vent, par rapport à ceux des îles Australes, est liée à la

ses conditions d'accessibilité, de la plupart des puta tupuna qui n'ont pas une vocation de diffusion extérieure et se transmettent à l'intérieur du cercle restreint des descendants de leur rédacteur initial ou secondaire. Certaines récitations traditionnelles contenues dans ces Mémoires - publiés à ce jour uniquement en anglais (1922) et en français (1971) - ont d'abord été transcrites en tahitien par Marau Taaroa et figurent aussi dans différents dépôts d'archives (familiales et publiques).

8. Nous savons qu'un texte relatif à l'histoire des souverains de Mangareva, présenté comme ayant été rédigé par un élève du père Maigret, est publié (dans une traduction anglaise) à Honolulu dans les numéros des 12 et 19 décembre 1840 de l'hebdomadaire The Polynesian. En 1852, paraît également à Honolulu (à l'imprimerie de la mission catholique), un texte de 14 pages, en langue de Mangareva, que l'on dit avoir été conté en 1840 : E mau atoga Magareva akataito i kaia i Magareva ara e Matia Puputauki i te tau o te akariki, 1840 (récit de Mathias Puputauki sur les choses mangaréviennes arrivées à Mangareva au temps du roi, 1840). Les écrits du père Tiripone ont été rédigés alors qu'il se trouvait à Tahiti auprès du père Laval. Ces récits et manuscrits, inventoriés dans l'ouvrage de Laval (1938), sont pour la plupart conservés à Rome, aux archives centrales des pères de Picpus. 
question foncière. Annexées en 1900 à l'ensemble français, les îles de Rurutu et Rimatara (aux Australes) n'ont pas connu la même législation sur la propriété privée que les îles du-Vent et Sous-le-Vent. À Rurutu et Rimatara, ce qui s'est produit à Tahiti dès les années 1852, l'enregistrement de déclarations de propriété foncière dites tömite (du nom du "comité » chargé de les recueillir), n'a pas eu lieu. Dans ces deux îles des Australes, la preuve de la propriété foncière s'est faite, jusqu'à la deuxième moitié $\mathrm{du} \mathrm{Xx}^{\mathrm{e}}$ siècle, sur la base de la tradition orale, qui s'est trouvée confortée par l'existence de cahiers de tradition fixant par écrit généalogies et histoires de familles (notamment les chroniques des guerres passées, justifiant l'appropriation de tel ou tel territoire à Rurutu); d'où une motivation particulière des habitants de cette île pour l'élaboration puis le recopiage des plus anciens puta et leur transmission jusqu'à ce jour. Au moment où le cadastre de Rurutu fut établi, au début des années 1950, des registres familiaux consacrés aux terres (répertoriés par Alain Babadzan comme puta fenua ${ }^{9}$ - ou puta'епиа -) apparurent afin de conserver la mémoire des conseils de famille et autres procédures d'attribution de droits fonciers (certaines pouvant dater d'avant le cadastrage proprement dit et trouver leur place à l'intérieur de ce nouveau type de registres).

L'existence de cahiers consacrés aux affaires de terres, à côté des puta tupuna " historiques" est assez spécifique à Rurutu. Ailleurs, les informations relatives aux terres figurent à l'intérieur des puta tupuna et y font rarement l'objet d'une section particulière. Ces textes témoignent en effet davantage du souci de conserver la mémoire de personnages ou événements anciens, qui risquent de se perdre, que celle de noms de lieux qui ne sont évoqués que par rapport aux hauts-faits de ces personnages.

D'autres raisons locales expliquent que soient particulièrement connus en Polynésie française les puta tupuna de Rurutu, du moins des chercheurs et des gens ayant accès aux bibliothèques ou aux photocopieuses. Il s'agit surtout de la présence à Rurutu, dans les années 1950, de l'Américain Martin Brunor, installé sur place et passionné de l'histoire ancienne de l'île. Brunor a constitué un fonds d'archives unique, sous forme de textes dactylographiés, de photos ainsi que d'originaux de puta tupuna qui lui avaient été confiés par des petits notables de l'île ${ }^{10}$ pour être sauvegardés. Il les avait convaincus que leurs cahiers traverseraient mieux le temps en Amérique, dans un musée. Ainsi, autour de 1960, le pasteur Teriimana Poetai et d'autres détenteurs de traditions de Rurutu lui ont confié certains cahiers ancestraux, aujourd'hui entreposés aux archives du Peabody Essex Museum de Salem dans le Massachusetts ${ }^{11}$. Ils en obtinrent en échange des copies et procédèrent pour euxmêmes à un recopiage ${ }^{12}$ avec parfois ajout à ces textes de développements contemporains, conduisant aux altérations que nous avons mises en évidence à propos de l'assimilation de certains ancêtres de l'île à des "Peaux-rouges » ou « Incas » (Saura, 2005a).

Dans une texte général, Bibliography of the Polynesian Manuscripts at the Peabody Museum of Salem ${ }^{13}$, Martin Brunor explique que le premier manuscrit de Rurutu aurait été écrit par le roi Paa Teuruarii dans la deuxième moitié du $\mathrm{XIX}^{\mathrm{e}}$ siècle et que c'est de cet ouvrage que seraient inspirés la plupart des puta tupuna conservés à Salem. Martin Brunor ajoute ne pas avoir luimême eu en main l'original de ce puta tupuna initial, qu'il chercha jusqu'à Londres où Paa Teuruarii aurait vécu trois ans. Ces informations sont à prendre avec circonspection. Aucune autre source - et notamment aucun puta de Rurutu à notre connaissance - ne mentionne un quelconque séjour de Paa en Angleterre. C'est peut-être parce sa mémoire est étroitement associée à l'œuvre des missionnaires anglais qu'un tel voyage est posé, de même que, noblesse oblige, c'est à lui qu'est attribuée l'écriture du premier puta de Rututu - dans lequel les suivants ne sauraient que prendre source.

L'entreprise de sauvegarde des puta tupuna de Rurutu par Martin Brunor et les travaux ethnologiques de Pierre Vérin (1969) et d'Alain Baba225).

9. La distinction entre ces cahiers et les puta tupuna proprement dits n'est toutefois « pas absolue » écrit-il (Babadzan, 1979 : 10 .

10. Néanmoins, des éléments de puta tupuna de Rurutu sont connus bien avant l'entreprise de sauvegarde de Martin Brunor. Ainsi, un texte E parau tupuna no to matou fenua, no Rurutu, traduit en anglais par Teuira Henry, est publié dès 1911 dans le Journal of the Polynesian Society (pp. 136-139).

11. En particulier, le manuscrit appartenant à Tamaiti Rita Tepa (dont l'écriture se clôt en 1882); celui du pasteur Taputu Mateau également fort ancien (lui-même est décédé en 1947 à près de quatre-vingt-dix ans); celui du régent et haut-juge Natapu Hurahutia (dont la partie généalogique est datée de 1890, suivie de textes allant jusqu'aux années 1940) ; celui de Maimoa Mairau ; celui de Nootu Manate.

12. Au sujet du recopiage et de la réécriture des traditions populaires du Moyen Âge (qu'il distingue, par une volonté de fidélité, de celui des traditions religieuses), Zumthor note que, de toute façon, s'agissant d'une société à tradition orale, «l'idée même de copie paraît trop moderne : foncièrement, le manuscrit est re-création » (1987 : 114-115).

13. E-12, Martin Brunor's papers, James Duncan Phillips Library, Peabody Museum, Salem. 
dzan (1979), menés en partie sur la base des manuscrits de cette île, ont donc contribué à conférer une publicité particulière aux manuscrits de cette île. Ajoutons qu'en 1997, un originaire de Rurutu, Daniel Terooatea, a publié à compte d'auteur sous le titre Te va'a ta'ata mātāmua $i$ tapae $i$ Rurutu (Les premières pirogues arrivées à Rurutu) des éléments (partiellement réécrits) d'un puta tupuna de sa famille, sous la forme d'un ouvrage (entièrement en langue vernaculaire) de 191 pages. Le titre renvoie à des traditions relatives au peuplement de l'île, sur lesquelles s'ouvrent certains puta tupuna de Rurutu, dont celui de Puna Taputu - dit Puna Haava (que nous connaissons et dont est proche le texte de Daniel Terooatea) ${ }^{14}$.

Enfin, sur l'île de Rurutu, depuis une dizaine d'années, un certain nombre de personnes âgées se regroupent au sein de la structure des To'ohitu ${ }^{15}$ pour travailler à l'écriture d'une histoire de l'île, sur la base des différents puta tupuna de chaque famille. L'espoir que l'ensemble de ces récits - souvent contradictoires - formant la tradition orale de l'île pourrait fusionner pour donner naissance à une version unique et définitive de celle-ci est évidemment illusoire : cette version nouvelle ne ferait que s'ajouter aux autres, sans les faire disparaître. Cette illusion procède inconsciemment de la sacralisation de l'écriture évoquée précédemment, dans certains milieux protestants polynésiens. Ainsi, la tradition orale, à l'image de la parole de Dieu, ne saurait être qu'une : fondamentalement parau mau, dans le double sens de parole vraie et de parole fixe (fixée par l'écrit), parole vraie parce que fixée dans le Livre.

\section{Deux puta tupuna des îles Sous-le-Vent}

Hormis les puta tupuna des Australes, ceux des îles Sous-le-Vent sont depuis peu l'objet d'une attention et d'une publicité accrues en Polynésie française. Cela est dû à l'impulsion donnée à leur publication par l'écrivaine et linguiste tahitienne (en fait, originaire de Huahine) Louise Peltzer. Universitaire (elle accède en 2005 aux fonctions de présidente de l'université de la Polynésie française), elle fut de 1998 à 2004 ministre de la Culture du gouvernement de la Polynésie française. En 1999, Louise Peltzer décidait de créer à Tahiti la série des Cahiers du patrimoine au sein desquels seraient publiés des manuscrits traditionnels. Le nom tahitien choisi pour cette collection est Puta tumu (livre originel/fondamental/souche) et non Puta tupuna. Ce dernier intitulé pouvait donner l'impression que seraient portées dans la sphère publique des informations de nature familiale, privée (tupuna désignant les ancêtres). Néanmoins, les appellations puta tupuna et puta tumu se rejoignent très largement et sont très interchangeables.

Le premier texte publié est un manuscrit datant de 1846, accompagné d'une traduction et d'une édition critique (Saura et al., 2000) sous le titre Histoire et traditions de Huahine et Porapora. Le deuxième (Saura et al., 2003), datant des années 1910, édité sous une forme assez analogue, a pour titre La dynastie des Tama-toa de Ra'iātea (îles Sous-le-Vent). Puta 'à'amu nō te 'ōpū ari'i Tama-toa nō Ra'iātea ${ }^{16}$ (" livre de récits de la dynastie des Tama-toa de Ra'iātea »). Nous allons revenir séparément sur chacun de ces manuscrits qui s'avèrent d'une richesse particulière à l'analyse et illustrent la variété du " genre » puta tumu (ou puta tupuna).

\section{Histoire et traditions de Huahine et Porapora}

Ce manuscrit nous est parvenu en 1998 sous la forme d'une photocopie du dernier recopiage (effectué en 1963) d'un texte original datant de 1846. La trentaine de pages du document manuscrit s'est transformée en une édition critique de 89 pages comprenant une introduction, la reproduction dactylographiée du texte original, une nouvelle version de ce texte avec systématisation scientifique avec des glottales et des longueurs, une traduction et un appareil de notes.

14. La même année (1997) avait paru, sous la plume d'une habitante de Rurutu, Pare Teinaore Walker, un article au titre et au contenu très proches ('Áai nō te mau táata mätāmua i tae i Rurutu). Il figure dans l'ouvrage collectif Hei Pua Ri'i publié par l'académie tahitienne sur la base des textes des concours littéraires de cette institution. Ce texte de Pare Teinaore Walker est largement réécrit, présentant une forme littéraire contemporaine qui n'est pas celle, plus sèche et simplement informative, des puta tupuna anciens. Une version carrément romancée de cette même histoire de migrations apparaît dans un ouvrage ultérieur (1999) de la même écrivaine ; voir nos commentaires dans Saura (2005a).

15. Autrefois constituée de juges d'appel, notamment spécialistes des questions foncières. Cette structure judiciaire a disparu en 1945 mais a revu le jour à la fin du $\mathrm{Xx}^{\mathrm{e}}$ siècle sous une forme associative, remplissant désormais essentiellement une fonction culturelle. Au sujet des To'ohitu, voir Saura (1995).

16. Entre ces deux textes s'intercale (2001) le Cahier du patrimoine 2 Porapora i te fänau tahi. Mille ans de mémoire (Borabora la première née), qui ne fait pas partie de la collection Puta tumu. Il ne s'agit pas d'un puta tupuna mais d'un texte rédigé aujourd'hui par une historienne tahitienne, Martine Ratinassamy, principalement sur la base du Tahiti aux temps anciens de Teuira Henry, d'enquêtes orales et d'un puta tupuna des îles Sous-le-Vent conservé au service de la Culture et du Patrimoine de la Polynésie française (ex-Centre polynésien des sciences humaines). 
Rédigé par un Polynésien des îles Sous-le-Vent (hélas non identifié), ce texte relate une période cruciale de l'histoire de deux de ces îles, Huahine et Borabora, marquée par l'arrivée des premiers navires occidentaux et l'installation des missionnaires anglais. Les événements qu'il aborde se déroulent des alentours de 1770 au tout début des années 1820 . Il ne traite pas du mouvement anti-missionnaire Mamaia de la fin des années 1820, ni de l'intrusion politique française dans la région une décennie plus tard. Ce petit livre se veut avant tout une histoire des chefs (ari'i $)^{17}$ et guerriers ('aito) illustres de ces îles ainsi que des batailles qu'ils menèrent jusqu'à leur conversion au protestantisme. L'auteur salue l'abandon de la religion traditionnelle particulièrement exigeante en vies humaines, au profit du christianisme, religion de la paix. Toutefois, il ne fait pas des missionnaires des héros, concentrant toute son attention sur la personne des ari'i (chefs), tentant d'expliquer les liens familiaux qui les unissent, s'attardant sur leurs qualités ou leurs défauts, leur bravoure ou leur cruauté.

La chronologie des événements qu'il livre est souvent inexacte en comparaison du contenu des correspondances missionnaires qui relatent des mêmes événements. Ses informations généalogiques présentent également une certaine fantaisie. Toutefois, ces erreurs (avérées s'agissant de personnages $\mathrm{du} \mathrm{XVIII}^{\mathrm{e}}$ siècle dont l'identité est connue) ne nuisent pas réellement à la valeur de ce document, qui réside ailleurs : précisément dans le fait de disposer d'une écriture autochtone fort ancienne, que nous pouvons aujourd'hui analyser avec du recul.

On peut s'étonner de la similarité de certains passages du texte avec d'autres du Tahiti aux temps anciens de Teuira Henry (publié pour la première fois en 1951) élaboré sur la base des traditions recueillies par son grand-père, le missionnaire John Orsmond. Il y a tout lieu de croire que l'auteur avait appris les mêmes récitations que les informateurs d'Orsmond qui vivaient également dans ces îles durant les décennies 1820 et 1830 . Ce puta tupuna, très ancien, est sans doute celui qui révèle le plus d' ' indices d'oralité » pour reprendre l'expression de Zumthor (1987 : 37). Peut-être convient-il mieux d'évoquer ce texte à l'aide du concept de "manuscriture » que d' " écriture » proprement dite, suivant le même Zumthor (1987 : 110) qui, partageant les remarques de Marshall McLuhan (1967) et de Walter J. Ong (1982), évoque certains textes européens anciens comme "peuplés par des bruits [analogues à ceux] parasitant la communication orale... Il nous arrive souvent de percevoir dans le texte la rumeur, éclatante ou confuse, d'un discours parlant de la voix même qui le porte $»$.

Sur le fond, l'apport de l'ouvrage tient parfois à certains détails apparemment mineurs surgissant au détour des pages mais incitant à l'approfondissement. Nous en donnerons un exemple à travers l'usage récurrent du chiffre huit sous la plume de l'auteur. Ainsi, l'île de Borabora estelle présentée comme divisée en huit mata'eina'a (chefferies) réunies sous la domination de la vallée de Fa'anui. Au XIX ${ }^{\mathrm{e}}$ siècle, ces chefferies ont à leur tête huit gouverneurs (tāvana) dont les noms sont également énumérés. Sont ensuite évoqués huit 'ōpū matahiapo (ramages aînés) ou principales lignées de chefs. Enfin, lorsque l'auteur se remémore les grands chefs traditionnels et guerriers de cette île, il en répertorie exactement huit, pas un de plus.

Ce découpage à l'aide du chiffre huit repose sur des réalités sociologiques et culturelles effectivement très polynésiennes. On retrouve en effet la même catégorisation des groupes sociopolitiques dans d'autres îles comme par exemple à Tahiti à travers l'alliance des huit chefferies (mata'eina'a) des Teva. En fait, même si le nombre exact de chefferies de ces îles, ou d'une partie de ces îles, varie la plupart du temps entre six et dix, les hommes s'arrangent pour considérer le chiffre huit comme la base, le chiffre pivot de l'organisation politique de l'île, autour duquel s'articulent éventuellement d'autres entités. L'auteur du puta tumu n'échappe donc pas à cette très polynésienne nécessité structurale d'ordonner la réalité sociale, voire même de transmettre la mémoire des hommes, sur la base du chiffre huit (voir Saura, 2005b : 74-75).

S'agissant de la langue d'écriture, on remarque surtout son utilisation de consonnes qui n'existent pas en tant que phonèmes du tahitien (comme le s) mais qui, en tant que lettres de l'alphabet, figurent néanmoins dans la traduction tahitienne de la Bible datant de 1836. Les missionnaires avaient choisi de les conserver afin de mieux restituer la racine hébraïque ou grecque de certains noms propres ou noms communs. Toutefois, à l'oral, ces consonnes sont prononcées par les Polynésiens suivant les phonèmes de leur langue : à Tahiti, les lettres $\mathrm{k}, \mathrm{z}, \mathrm{s} . .$. sont lues comme un $\mathrm{t}, 1$ comme un $\mathrm{r}$, b comme un $\mathrm{p}$, etc. Formé à l'école de l'écriture protestante en langue tahitienne, l'auteur de ce puta tumu uti-

17. Nous traduisons ari'i par chef, pour les temps antérieurs au XIX ${ }^{\mathrm{e}}$ siècle. Ensuite, avec l'apparition de royautés centralisées, «à l'européenne », c'est le terme roi qui est employé dans toutes les sources officielles pour évoquer les ari'i. Par exemple, les États du protectorat tahitien (1842-1880) sont aussi connus sous les appellations Royaume tahitien ou Royaume des Pōmare. 
lise à juste titre ces consonnes absentes de sa langue lorsqu'il emploie des néologismes : sabati pour sabbat "dimanche », buta pour livre (de l'anglais book). Mais il a parfois tendance à recourir abusivement à ces consonnes étrangères pour transcrire un néologisme qui ne les nécessiterait pas. Par exemple, 'etene (païen, préchrétien) est écrit esene alors qu'il s'agit là d'une adaptation phonétique de l'anglais heathen (Montillier, 1999 : 33) qui devrait s'écrire tout simplement etene ou 'etene. De même, bapetito (baptiser), qui pourrait s'écrire bapetizo en suivant la graphie biblique, devient sous sa plume babetito, avec une transformation phonétique du $\mathrm{z}$ en $\mathrm{t}$ comme dans la langue orale mais aussi un recours injustifié au deuxième b là où il conviendrait d'utiliser le $\mathrm{p}$ tahitien ordinaire. Cette tendance excessive à utiliser des lettres bibliques absentes du système phonologique tahitien se retrouve dans la plupart des textes vernaculaires rédigés au $\mathrm{XIX}^{\mathrm{e}}$ siècle. Elle exprime une volonté appuyée de se rapprocher de la langue des missionnaires mais aussi du grec et de l'hébreu, de la langue du texte biblique. Précisément, l'auteur de ce manuscrit n'est animé par aucune nostalgie du passé pré-chrétien. Il dresse, à l'inverse, un tableau manichéen des méfaits de l'ordre religieux ancien et des bienfaits de la conversion et achève son manuscrit par un glorieux « amen ».

On est loin de pouvoir employer ici l'expression de « vision des vaincus » rendue célèbre par les travaux de Nathan Wachtel consacrés aux sociétés andines au $\mathrm{XVI}^{\mathrm{e}}$ siècle. Dans ce puta tumu, il n'est pas question, du point de vue de l'auteur, de défaite devant les étrangers mais d'une acculturation volontaire et salvatrice. Le changement religieux ne se confond pas, il est vrai, avec la colonisation politique, qui a lieu ultérieurement.

\section{La lignée royale des Tama-toa de Ra'iātea}

Relatif à une période historique plus étendue, qui comprend la colonisation (politique), est le puta tumu publié en 2003 sous le titre Puta 'à'amu nō te 'ōpu ari'i Tama-toa nō Ra'iātea (La lignée royale des Tama-toa de Ra'iātea - îles Sous-le-Vent). Ce manuscrit en langue tahitienne, dont on ignore l'auteur, a été retrouvé par nous au Service territorial des archives de la Polynésie française, sous une forme dactylographiée. Il était tombé dans l'oubli, si l'on excepte une tentative de traduction en anglais en 1982 par un résident de Tahiti (Ralph White), qui n'a pas donné lieu à publication. Une autre version dactylographiée du texte original (quasiment identique, à quelques lettres près) figurait aux archives de l'Église évangélique de Polynésie française, ce qui montre que ce document avait déjà suscité l'intérêt de personnes désireuses de le présenter sous une forme très lisible.

Ce texte se veut une histoire intérieure, intime, de la dynastie des Tamatoa de Ra'iātea, s'attachant tout particulièrement à la période $\mathrm{du} \mathrm{XIX}{ }^{\mathrm{e}}$ siècle. Il paraît avoir été rédigé en deux temps, par deux membres de cette famille. Dans ses premières pages figure la mention «Vairao le 24 juin $1874 »$ : il est donc très probable qu'il ait commencé à être rédigé à Vaira'o (presqu'île de Tahiti), par un membre de la famille Tamatoa, peut-être par un fils du Tahitoe, personnage clé du livre, qui eut une descendance dans cette partie de Tahiti. Cette proximité avec Vaira'o ne concerne pas la seule période contemporaine puisque le texte s'ouvre par une généalogie fort ancienne précisant la nature des liens entre la lignée des Tamatoa de Ra'iātea et celle des chefs Vehiatua de la presqu'île de Tahiti.

La première partie du texte est brève et uniquement d'ordre généalogique. La suite, c'est-àdire la majeure partie du document, épouse la forme d'un récit historique qui, pour l'essentiel, s'arrête aux environs de 1888. Nous pensons qu'il a pu être rédigé par Te-ari'i-nui-o-Ra'i-ātea a Tahitoe et/ou par son fils 'U'uruari'i-i-Tainuu a Tahitoe, à la fin du XIX ${ }^{\mathrm{e}}$ siècle ou au début du $\mathrm{XX}^{\mathrm{e}}$ siècle. Le fait que l'ouvrage ait été écrit en plusieurs temps est également suggéré dans les dernières lignes du manuscrit : celui-ci se clôt sur le récit de la découverte, par des membres de la famille Tamatoa, dans les années 1880, d'un manuscrit rédigé par celui que l'auteur nomme alors Tamatoa IV et qui, partout dans le reste du texte, était présenté comme Tahitoe, fils de Tamatoa IV.

Ce problème de numérotation (de prosopographie) nous a conduit à de longs développements dans la préface que nous avons donnée à cet ouvrage, à travers une comparaison de différentes versions publiées de la généalogie des Tamatoa, à laquelle s'ajoute désormais celle de l'auteur de ce puta tumu.

Ce manuscrit, à la forme narrative presque romancée, offre une version assez approximative de l'histoire, tant pour ce qui est de la chronologie que s'agissant des noms des personnages. Mais son intérêt réside précisément dans le fait que cette écriture autochtone est une reconstitution, une reconstruction a posteriori qui se veut cohérente là où les hommes et les événements ne l'ont pas toujours été. Elle tend à simplifier les situations. Elle vise aussi à donner sens à des événements ; à assigner une fonction à des per- 
sonnages, qui n'était pas exactement la leur à leur époque mais qui après coup explique, rationalise leurs actions. Le meilleur exemple en est la justification du nom du personnage central du texte, le roi Tahitoe des années 1870 . L'auteur pose que ce nom aurait été choisi par son grandpère le roi Tamatoa III (dit Tamatoa le grand), avec la signification suivante : un/le (tahi) roi qui demeure/reste (toe), c'est-à-dire que Tahitoe serait le dernier roi de cette lignée. En fait, cette étymologie est discutable ${ }^{18}$, mais surtout, d'autres personnages de la famille ont porté ce nom avant lui, ce qui compromet la signification particulière que lui donne l'auteur. Tahitoe est dans son texte le dernier roi acclamé sur le marae Vaeara'i, le dernier roi d'une île souveraine, celui à qui le destin assigne la double tâche d'accueillir l'évangile et de remettre la royauté de Ra'iātea aux mains des Blancs. Tout cela, le grand-père l'a prédit, de même que les tahu'a (prêtres et oracles) du dieu Ta'aroa.

Une telle anticipation de l'histoire nous rappelle la célèbre prophétie de Vaitā, grand prêtre à Ra'iātea, censé avoir prédit l'arrivée des premiers hommes blancs et des missionnaires avant même le passage de Wallis à Tahiti en 1767. Les circonstances historiques de l'émission de la prophétie de Vaitā ont été analysées par H. A. H. Driessen dans un excellent article (1984). Selon lui, la prophétie de Vaita pourrait avoir été émise avant 1767 sur la base d'informations qui circulaient déjà au sujet du passage de navires occidentaux présents dans la région depuis au moins cent cinquante ans. Nous pouvons y voir aussi une rationalisation a posteriori, l'événement historique que constitue l'arrivée des Européens donnant lieu à cette prophétie à naître après coup, parce qu'il faut bien donner un sens à l'événement, lequel est toujours, dans une société traditionnelle, rapporté au mythe ou perçu comme la volonté des dieux.

Il en va de même avec la célèbre « transe » de Ari'ipaea vahine, reine de Huahine, épouse de Pomare II et fille de Tamatoa III le grand, « transe » qu'évoque Teuira Henry dans Tahiti aux temps anciens (1951 : 228-230). La scène est dite se dérouler à Huahine " au début du XIX siècle, peu avant l'installation du christianisme ", c'est-à-dire à un moment où l'on sait qu'il existe une nouvelle religion mais avant que celle-ci ait été adoptée. Ari'ipaea vahine tomba donc dans un profond sommeil qui dura un mois, au cours duquel son esprit voyagea. Elle s'éprit d'un esprit de Ra'iātea, qui, au moment où elle allait reprendre son enveloppe corporelle, lui « déclara qu'il continuerait à venir la voir [...] à condition toutefois qu'elle n'embrasse pas la religion chrétienne, comme le faisaient beaucoup de Tahitiens [...]. Pendant un ou deux ans après son rétablissement, elle communiqua souvent avec son amant esprit, jusqu'au jour où s'étant rendue à Moorea, elle rencontra des Tahitiens qui lisaient et étudiaient les Saintes Écritures et se joignit à eux [...]» (ibid.). C'est alors que l'esprit lui apparut pour la dernière fois, conformément à ce qu'il lui avait annoncé.

Au sujet de la naissance de Tahitoe dans cet ouvrage, comme de la prophétie de Vaitā ou de la " transe de Ariipaea vahine », nous proposons d'avancer le concept d'épisode historique total, à la façon dont on parle avec Marcel Mauss de « fait social total ». Ces épisodes remplissent en effet pour leurs protagonistes et ceux qui en conservent la mémoire, une fonction essentielle : celle de faire accepter la nécessaire renonciation au monde des esprits et des divinités ancestrales ; également, dans le cas de Tahitoe, la nécessaire disparition de la royauté indigène. Comme Vaitā et comme Ari'ipaea vahine, le roi Tahitoe, personnage central du texte La lignée royale..., est la figure charnière du passage d'un univers à un autre. D'ailleurs, dans ce manuscrit, à chacun des personnages de la lignée des Tamatoa, une fonction est impartie : celle de Tahitoe est essentiellement politique, tandis que son grand-père Tamatoa III incarne la résistance à l'acculturation religieuse et que son père Tamatoa IV est celui qui réussit la mise en place des codes de lois missionnaires. Cette restitution synthétique présente à l'évidence de sérieux écarts avec l'histoire qui nous est rapportée par les missionnaires ayant vécu cette période au quotidien.

C'est enfin et justement sur la manière dont il offre une version conciliante de l'histoire de Ra'iātea-Taha'a que ce texte est le plus étonnant. Il minore les prétentions au pouvoir des chefs autres que les Tamatoa, seuls dotés de légitimité aux yeux de l'auteur. C'est faire l'impasse sur une donnée fondamentale de la structure du pouvoir politique aux îles de la Société, à savoir l'existence de plusieurs chefs sur un même territoire ou une même île, l'un étant titulaire des droits dynastiques, l'autre exerçant la réalité du pouvoir militaire, du gouvernement. Cet équilibre précaire des pouvoirs entre le chef titulaire et les chefs tribaux a bien été étudié par Douglas Oliver (1974, vol. 2) au sujet du Tahiti du XvIII ${ }^{\mathrm{e}}$ siècle et Jean-François Baré (1987) à propos des îles Sous-le-Vent au $\mathrm{XIX}^{\mathrm{e}}$ siècle. Le présent manuscrit donne des informations en ce sens

18. Voir, suite à notre introduction (2003: 3-27) la note relative aux descendants des lignées Tamatoa, Pehupehu et Tahitoe (2003 : 28-37) du juge René Calinaud. 
lorsque son auteur évoque à l'intérieur d'une même chefferie (mata'eina'a) la lignée des aînés (ari'i) détenteurs de titres et la lignée des cadets à qui revient l'exercice, par délégation, de fonctions d'encadrement politique. Toutefois, il néglige l'influence politique réelle de ces lignées cadettes, réservant la part belle aux seuls aînés (ari'i) dont le pouvoir, d'essence divine, n'est jamais susceptible d'être mis à mal.

Il est d'autres points essentiels sur lequels ce manuscrit "parle» considérablement par ses silences : son auteur, ne disposant d'aucune culture relative aux guerres traditionnelles, omet la dimension guerrière de ces îles et réduit les activités des ari'i à des discours, déplacements, fastes. Politiquement, il choisit aussi de minorer l'importance historique de la lignée TamatoaPōmare (de Tahiti) pour évoquer la seule branche des Tamatoa-Tahitoe (résidents de Ra'iātea), à laquelle il appartient très vraisemblablement.

Il convient donc au lecteur de savoir lire entre les lignes ; d'apprécier ce manuscrit pour ce qu'il apporte sur le fond, comme pour la belle langue dans laquelle il est écrit. Sa lecture permet aussi de réaliser qu'un puta tumu ou puta tupuna n'est jamais un simple recueil de traditions acceptables par tous et que les plus récents d'entre eux, ceux écrits hors du contexte de la transmission de la tradition orale, se situent parfois aux confins du roman historique. Est-il encore approprié dans ce sens, d'évoquer le texte $L a$ lignée royale des Tamatoa... en tant que puta tupuna ou puta tumu? Nous croyons que oui car, outre le fait que le sujet dont il traite est relatif aux anciens Polynésiens, ce manuscrit n'emprunte pas à d'autres sources que des traditions familiales ou des écrits familiaux antérieurs. Il se différencie en ceci des traditions contées par Tearapō sur les ondes de Radio Tahiti entre 1962 et 1964, publiées par le Centre polynésien des sciences humaines (Tearapō, 1997, 1999, 2000a, 2000 b, 2000c, 2000d), dans lesquelles il est aisé de constater les emprunts à des écrits imprimés, notamment à l'ouvrage Tahiti aux temps anciens de Teuira Henry.

\section{Les prétendus livres des ancêtres d'aujourd'hui. L'œuvre de Charles Manutahi}

Nous voudrions terminer cette évocation des manuscrits autochtones de Polynésie française par celle des œuvres de l'écrivain tahitien Charles Manutahi, qui prétendent au statut de récits traditionnels alors qu'il s'agit parfois de savoirs polynésiens réinterprétés à l'aune d'ouvrages ésotériques occidentaux contemporains. Cette précision pourrait suffire à les disqualifier, mais il convient de s'y attarder en raison de la circulation de ses textes dans une Polynésie française au lectorat aujourd'hui avide de savoirs traditionnels et qui mérite sur ce point une mise en garde.

Né à Taha'a dans les années 1940 d'une famille originaire des îles Sous-le-Vent, Charles Manutahi a été élevé à Mo'orea et en NouvelleCalédonie. Bon orateur en langue tahitienne, c'est aussi un fervent lecteur de la Bible. Nous présenterons ici pour commencer deux de ses livres (1982 et 1997) assez proches dans leur contenu de véritables puta tupuna. Puis, nous en examinerons deux autres (1992 et 2005) relevant d'une écriture ésotérique polynésienne très personnelle.

En 1982, paraît à compte d'auteur ${ }^{19}$ son premier ouvrage : Contes et légendes de la Polynésie. Le secret des livres tupuna. Te parau huna a te mau tupuna. Ce texte se compose d'une suite de légendes de Borabora et Tahiti (Heitarauri, l'enfant élévé par des requins; le fabuleux chien Piihoro...), auxquelles s'ajoute un texte relatant une réunion de varua'ino (esprits malfaisants) sur un îlot de Borabora en 1981. Ce livre de 173 pages, bilingue, se veut un résumé en tahitien moderne (1982 : 91-173) précédé de sa traduction en français (1982 : 1-90) de ce que Charles Manutahi se souvient avoir lu dans le puta tupuna d'un de ses oncles (personnage photographié, sans indication de son identité), qui aurait été enterré avec son manuscrit.

Publié en 1997, Te parau o Papenoo, e peho no Tahiti. L'histoire de la vallée profonde de Papenoo, île de Tahiti comprend des traditions relatives à cette vallée ainsi qu'à la déesse Pere qui lui est liée et dont l'histoire s'étend jusqu'à Fakarava (Tuamotu) et aux îles Hawaii et, également, quelques traditions de Borabora. L'ouvrage s'ouvre là encore sur un texte en français (1997 : 1-73) qui s'avère la traduction du texte en tahitien qui suit (1997 : 74-142), présenté comme un ensemble de légendes issues du puta tupuna ${ }^{20} \mathrm{de}$ Tehaamarumaruarii a Manutahi, de Borabora. Ce puta tupuna aurait été écrit (1997:69) « dans les années 1820 à Vavau » (ancien nom de Borabora). Cette dernière information est sujette à caution, car si l'apprentissage de l'écriture par les Polynésiens des îles Sous-le-Vent débute bien à cette période, il est très douteux qu'un manuscrit ait été rédigé aussi tôt.

Du récit de traditions anciennes à l'interprétation personnelle, la démarche est franchie par

19. En fait, aux éditions Te hiroa maohi tumu (La culture polynésienne originelle), que fonde alors Charles Manutahi.

20. Selon ses ouvrages, l'auteur emploie indifféremment puta tupuna ou puta tumu pour mentionner ce manuscrit. 
Charles Manutahi en 1992 avec la parution d'un ouvrage au titre ambitieux et énigmatique : Te parau huna o te ao mä'ohi. Le mystère de l'univers $m \vec{a}$ ohi. Sur la couverture est reproduit le dessin tracé dans les années 1860 par le chef régent Paiore de l'île de 'Anaa (Tuamotu), représentation cosmogonique d'un univers stratifié comprenant un monde des hommes et un monde des dieux. L'auteur se propose dans son texte d'expliquer chacun des signes figurant sur ce dessin.

Cet ouvrage ne saurait laisser indifférent, à commencer par son titre. On peut tout d'abord s'interroger sur l'expression te parau huna o te ao $m \bar{a}$ 'ohi : si véritablement parole(s) enfouie(s) (parau huna) il y a, comment Charles Manutahi réussit-il à y accéder et, ce faisant, respecte-t-il la volonté de ceux qui auraient pu souhaiter que ces traditions demeurent secrètes? La dénomination Te ao mā'ohi (l'univers polynésien) n'est quant à elle pas traditionnelle mais fleure bon la pensée identitaire et l'idéologie indépendantiste de la fin $\mathrm{du} \mathrm{Xx}^{\mathrm{e}}$ siècle ${ }^{21}$.

Pour comprendre sur la base de quelles informations a été rédigé cet ouvrage, il faut lire sa quatrième de couverture ainsi que les indications (pp. 105-113) figurant à la fin du texte principal bilingue. Elles viennent :

- Pour une part, des traditions issues du puta tupuna de Tehaamarumaruarii a Manutahi des îles Sous-le-Vent. Charles Mautahi précise (1992: 105) que dans ce manuscrit figurait l'indication que «le prince Huru manu a Manu tahi », arrière-grand-père de l'auteur, «était le dernier grand prêtre à posséder encore la connaissance des dieux. Il fut celui qui arrêta la connaissance du peuple mā'ohi $[\ldots] \gg »^{22}$.

- D'autre part, des informations recueillies auprès du grand-père de la femme de Charles Manutahi, nommé Kaivero Marama a Tehau, originaire des atolls de Takume, Raroia, Makemo et Hao (Tuamotu).

- Enfin, l'auteur explique s'être inspiré des écrits du colonel James Churchward $(\mathrm{Mu}$, le continent perdu. L'univers secret de Mu. Le monde occulte de $M u$ ), ce qui permet d'emblée de situer Te parau huna... à l'intérieur d'un type de littérature bien particulier, relevant du symbolisme et de l'ésotérisme.

Sur le fond, le dessin de Paiore, que Charles Manutahi commente sans distance critique dans son ouvrage, a pourtant déjà fait couler beaucoup d'encre. On sait l'enthousiasme qu'il avait procuré à la fin des années 1920 à Frank Stimson (ethnolinguiste de terrain) et toute la controverse qui l'opposa dans les années qui suivirent à Kenneth Emory au sujet du prétendu dieu Kiho que Stimson aurait découvert dans la tradition orale de certaines îles des Tuamotu. Stimson aussi s'était vu offrir par un nommé Paea de 'Anaa un schéma de l'univers traditionnel faisant étrangement écho à celui de Paiore.

$\mathrm{Au}$ sujet de la divinité Kiho, Stimson posait (1933a et 1933b) qu'elle n'aurait été connue que de rares initiés, à l'intérieur d'une religion ésotérique distincte de la religion " exotérique » des grandes divinités comme Tane ou Atea. Pour asseoir cette idée d'une primauté historique de Kiho sur les autres divinités polynésiennes; Stimson basait une partie de son argumentation sur le dessin du régent Paiore « recueilli » par Xavier Caillet autour de $1860^{23}$ et alors tout récemment reproduit ${ }^{24}$ dans l'ouvrage de Teuira Henry, Ancient Tahiti (publié en 1928 par le Bishop Museum de Honolulu). Or, Kenneth Emory s'est attaché à démontrer (1933, 1938, 1939, 1940a et b, 1943, 1947) que le schéma de Paiore n'était pas une représentation cosmogonique pré-chrétienne mais un dessin tardif, une recomposition initiée par Xavier Caillet et à l'élaboration de laquelle celui-ci avait même pris une grande part ${ }^{25}$.

Digne successeur d'un Stimson qui, influencé par ses lectures sur l'Égypte ancienne et l'hindouisme, se plaisait à trouver en Kiho une divinité originelle apportée par les plus anciennes vagues de peuplement de la Polynésie, Charles Manutahi fusionne pour sa part les théories du continent $\mathrm{Mu}$ avec des données relatives aux cultures amérindiennes et polynésiennes. Il affirme que la grande divinité cachée derrière le schéma de Paiore se nommerait Hau 'è (Hau, littéralement «paix, royaume, souffle» et 'e', « différent, séparé »). Hau 'ē, dont le nom signifie

21. Nous ne connaissons aucun texte traditionnel dans l'ouvrage de référence de Teuira Henry, Tahiti aux temps anciens où figurerait l'appellation totalisante Te ao mä'ohi (l'univers polynésien). Te ao mä'ohi est, en revanche, le nom choisi à la fin des années 1970 par le leader indépendantiste Oscar Temaru pour rebaptiser la Polynésie française ; il se rallie en 2005 au nom Tahiti nui proposé quelques années plus tôt par Gaston Flosse.

22. Il faut comprendre, celui avec qui s'arrêtèrent les connaissances pré-chrétiennes, ce qui est tout de même assez présomptueux.

23. Il existe plusieurs versions de ce schéma, dessinées entre 1859 et 1869 .

24. Voir pour l'édition française (1928 : 348) et (1951:356). Le schéma est accompagné d'un chant également transmis par Paiore, qui aide à sa compréhension.

25. Au sujet de cet officier de marine passionné de culture polynésienne, voir O’Reilly et Teissier (1962: 88-90). 
pour lui « Dieu qui a son royaume dans le ciel» (1992 : 30), aurait pour représentation symbolique un signe proche de la lettre Y. Il est présenté comme «le grand UNI» (1992: 31), porteur d'une unité culturelle première. Ces révélations apparaissent à Charles Manutahi au moyen d'un décryptage de chaque signe figurant dans le schéma de Paiore.

Le mythe d'une Polynésie connaissant autrefois une seule langue et culture ${ }^{26}$, unie par une même spiritualité, s'inscrit, à l'analyse, comme une représentation idéologique bien contemporaine. Il vise à abolir les frontières linguistiques et culturelles de cet ensemble d'archipels, au moyen d'un ésotérisme bon enfant, même si personne pas même Paiore, qui n'allait pas aussi loin - n'a jamais entendu parler d'une écriture symbolique de la cosmogonie polynésienne dont Charles Manutahi affirme pourtant dans ce livre détenir les clés.

D'une lecture toute aussi ébouriffante que le texte de 1992 est l'ouvrage Te parau o te mau vāhi faufa'a no te mau tupuna i Moorea. L'histoire des sites et des ancêtres de l'île de Moorea, paru en 2005. Ses sources principales d'inspiration revendiquées sont :

- des traditions provenant de son père adoptif Tetuarii Papai (de Afareaitu, Mo'orea) ;

- le manuscrit puta tumu de la famille Tehaamarumaruarii a Manu-Tahi ;

- des éléments de son expérience personnelle ;

- une enquête de cinq années à Mo'orea ;

- des sources livresques, à savoir, principalement les écrits de James Churchward (18521936) sur le continent $\mathrm{Mu}$ et Les Mémoires de Marau Taaroa (dernière reine de Tahiti).

L'affirmation centrale de l'ouvrage est (2005: 24, 101), qu'autrefois, un continent nommé Te ana feo pua raì émergeait sur l'océan Pacifique. Deux hautes pierres y furent installées par des prêtres dits Ramaiana (transposition polynésienne d'un élément de l'hindouisme ${ }^{27}$ ). Puis, ce continent s'effondra mais, grâce aux deux pierres qui jouèrent le rôle de piliers, subsistèrent des fragments de terre dont l'île de Mo'orea. Une de ces deux pierres était octogonale; l'autre avait une fonction symbolique, représentant ce qui donne la vie, c'est-à-dire, le nombril de la terre Ta'inuna $^{28}$ (Ta'inuna étant posé ici comme l'ancien nom de Mo'orea) ${ }^{29}$. Ces informations puisent bien aux racines de la mythologie polynésienne. Le «continent» en question s'apparente à la pieuvre mythologique dont les tentacules représenteraient les différents archipels du triangle polynésien, animal marin également très lié dans la cosmologie à l'île de Mo'orea. Les mythes tahitiens d'origine comprennent aussi nombre de récits mettant en scène des socles inférieurs et des socles supérieurs, ainsi que des traditions d'une solidarité primordiale des îles ${ }^{30}$ et du déluge (voir Teuira Henry, $1951: 462-473$ ) sans toutefois jamais faire état d'un continent proprement englouti (une idée directement puisée dans les traditions de $\mathrm{Mu}$ ).

La fusion de ces mythes polynésiens anciens et d'éléments mythiques venus d'ailleurs éclate dans le nom primitif supposé de Mo'orea, Taìnuna (ou Ta'inuna), que Charles Manutahi explique (2005: 24) par «Ua ta'i te hui tupuna $i$ to rātou fenua tei nū, i teie nei, ua nā rātou $»^{31}$ :

« Les ancêtres ont pleuré $\left(t a^{\prime} i\right)$ leur grande terre qui a été engloutie $(n \bar{u})$. Aujourd'hui, ils se sont calmés $(n \bar{a})$.»

Cette étymologie doit beaucoup à l'auteur car si les termes $\left(t a^{\prime} i\right)$ et $n \bar{a}$ existent bien en langue tahitienne avec la signification qu'il leur donne, en revanche, $n \bar{u}$ n'existe pas ${ }^{32}$. Ce $n \bar{u}$ est une « tahitianisation » d'un concept venu d'ailleurs, puisqu'on apprend plus loin que « dans le livre des morts, les Égyptiens ont nommé le continent englouti $\mathrm{Nu}$ et non $\mathrm{Mu}$. Les anciens Polynésiens lui ont également donné ce nom parce qu'il s'est enfoncé dans les profondeurs de l'océan » (2005: 63). Charles Manutahi avance à nouveau l'idée

26. Le fait que Paiore ait transmis des traditions cosmogoniques de l'atoll de Raroia n'est pas anodin car dans son ouvrage (1992), Charles Manutahi enrichit les traditions de Paiore de dires du grand-père de son épouse, également originaire de l'atoll de Raroia. On sait que dans cette île dont la population fut d'abord convertie (au milieu du XIX ${ }^{\mathrm{e}}$ siècle) par les Saints des derniers jours (mormons), des croyances relatives à l'usage d'une seule langue dans les Tuamotu d'autrefois, se sont mêlées avec le récit biblique de la tour de Babel et des éléments du Livre de Mormon, pour donner lieu à des élaborations étonnantes. (Voir Saura, 2004 : 149-151, 275-277).

27. Le nom Ramaiana n'est connu de personne en Polynésie. Il s'agit très clairement de la transposition par l'auteur du nom des épopées sacrées hindoues dites Rämäyana ( $\mathrm{V}^{\mathrm{e}}$ siècle avant Jésus-Christ, III $^{\mathrm{e}}$ siècle après Jésus-Christ) qui relatent la vie de Râma, incarnation de Visnu.

28. La symbolique du nombril d'une île est bien connue en Polynésie française. Voir Saura (2002).

29. Ce nom n'a jamais été répertorié nulle part, à notre connaissance, alors que 'Aimeho (également Eimeo), autre nom de Mo'orea, est très largement attesté, de longue date.

30. Dans les années 1820, William Ellis écrit : « Une de leurs traditions assure que toutes les îles étaient jadis unies en une fenua nui, une grande terre que les dieux détruisirent dans leur courroux, dispersant les morceaux dans l'océan » (1972 : 90 ).

31. C'est nous qui soulignons et mettons des italiques.

32. Il est absent du Dictionnaire de l'Académie tahitienne (1999), qui fait pourtant autorité en la matière. 
de l'existence d'un dieu premier, Hau 'è (Paix/ royaume spécifique), antérieur à Ta'aroa, et dont la connaissance se serait perpétuée jusqu'au moment des conversions au protestantisme dans les années 1810-1820. Précisément, une des figures mémorables de ce basculement des Polynésiens orientaux dans la religion chrétienne, le grand prêtre Pati'i de Mo'orea (connu pour avoir immolé en public ses objets de culte, défiant l'autorité des dieux ancestraux, en février 1815), aurait été un des ultimes détenteurs des savoirs secrets relatifs à Hau 'ē. Le marae 'Umarea de Afareaitu, desservi par Pati'i, aurait continué à incarner la continuité avec l'époque du continent $\mathrm{Nu}$. Charles Manutahi livre un croquis supposé être « extrait du manuscrit» (2005: 47), montrant trois pyramides - invisibles au profane supportant le marae 'Umarea qui « répétonsle, fut construit sur des bases triangulaires, celles de l'île même de Ta'inuna » (ibid.).

Le symbolisme du chiffre trois continue à être décliné au sujet d'un autre marae important de Mo'orea, le marae Nu'urua ${ }^{33}$ (au lieu-dit Varari, à Ha'apiti), avec l'affirmation (2005: 49-50) qu'il fut construit il y a plus de trois mille ans, à une période antérieure au dieu Ta'aroa, celle du dieu Hau 'ê, suivant des techniques de ciselage et de pose des pierres qui étaient celles « d'un autre peuple ». Cette datation imaginaire contredit les données scientifiques relatives au peuplement de l'ensemble tahitien (jamais envisagé comme antérieur à Jésus-Christ) mais, peu importe, car on entre ici dans l'ère de la croyance et le manuscrit s'avère en fait une réécriture syncrétique et personnelle du passé. Charles Manutahi écrit :

«Voici ce qui a été transcrit dans le manuscrit et dont je fais un résumé. Il y a trois mille ans, le dieu Ha'avare Huna prit le pouvoir sur les Ramaiana. Et il régna sur le pays et les îles tombèrent dans la nuit [...]. » $(2005: 60)$

Ainsi, Ta'aroa, rebaptisé Ha'avare Huna («Menteur masqué ») aurait-il supplanté Hau 'ē ; néanmoins, les adeptes du dieu primordial auraient réussi à en perpétuer la mémoire au moyen de pétroglyphes et de tapa (étoffes végétales) portant les symboles de sa grandeur passée et faisant également état de sa rivalité avec Ta'aroa. Un de ces symboles figure trois points suivis d'un signe proche de la lettre $\mathrm{Y}$ : c'est « le symbole de la nuit des temps, celui du Dieu Hau 'è et de la Trinité » (2005: 61).

Il faut comprendre ici qu'ainsi, la trinité chrétienne était préfigurée dans la religion de Hau 'ē, ce qui conduit aussi à poser une sorte de schéma historique faisant écho à celui que développe à la même époque l'écrivain et théologien tahitien Duro Raapoto (voir Saura, 1998) : dans les deux cas, la sévère religion de Ta'aroa est dite avoir remplacé une spiritualité polynésienne primordiale déclinée en termes de puissance et de paix. À l'analyse, on perçoit le poids de la culture chrétienne dans l'élaboration de ce scénario historique : à défaut de pouvoir trouver des vertus à la religion polythéiste et sacrificielle existant au moment de l'arrivée des Européens, ces auteurs se réfèrent à une prétendue première période de la religion tahitienne, sorte d'âge d'or ou de genèse océanienne qui leur permet de jeter malgré tout un regard positif sur une partie de la société des temps pré-européens.

L'idée d'une compatibilité intrinsèque de valeurs entre la religion des premiers Polynésiens et le christianisme n'est pas présentée comme une opinion de l'auteur mais comme une vérité pressentie par quelques rares sages au XIX ${ }^{\mathrm{e}}$ siècle. Ainsi, selon Charles Manutahi, c'est le grand prêtre Pati'i de Mo'orea qui aurait souhaité que le temple de Papeto'ai, premier temple chrétien bâti en dur dans les mers du Sud (dans les années 1820) ait une forme octogonale, en souvenir de la pierre octogonale soutenant autrefois l'île de Mo'orea. Sur les tombes de Pati'i et d'autres anciens tahu'a (spécialistes, prêtres) devenus diacres protestants, situées derrière le temple de Papeto'ai à Mo'orea, l'auteur explique (2005: 63) avoir retrouvé des signes de l'ancienne culture de $\mathrm{Nu}$ : deux colonnes rappelleraient les piliers du continent englouti, tandis que des triangles figureraient sa partie émergée.

$\mathrm{Au}$ total, cette synthèse personnelle de traditions empruntant à plusieurs cultures, dans une démarche relevant pleinement de l'ésotérisme, pourrait attirer la sympathie du lecteur si ce type de littérature s'affichait comme tel au lieu de prétendre révéler le contenu d'un manuscrit disparu. La fusion d'éléments des anciennes cultures d'Égypte, d'Asie, d'Amérique, ainsi que des théories du continent $\mathrm{Mu}$ ne peut évidemment provenir que d'un auteur contemporain et non d'un Polynésien du $\mathrm{XIX}^{\mathrm{e}}$ siècle. Il est donc particulièrement étonnant que ce livre soit présenté dans la préface qui lui est donnée, comme un texte à travers lequel Charles Manutahi « confirme ses talents de dépositaire d'un savoir ancestral. Il s'affirme à nouveau comme modèle dans une fidèle transmission de la mémoire des tupuna, d'une "oraliture" digne des écrits les plus savants » $(2005: 5)$. 


\section{Conclusion}

Au fil de cette étude, nous sommes passé progressivement de l'évocation de recueils de traditions puta tupuna rédigés en Polynésie française au $\mathrm{XIX}^{\mathrm{e}}$ siècle, à celle de manuscrits publiés aujourd'hui et présentés ou perçus comme les premiers même s'ils sont le fruit d'une écriture très contemporaine. En réalité, même les premiers manuscrits du $\mathrm{XIX}^{\mathrm{e}}$ siècle relèvent d'une reconstitution de la société traditionnelle dans la mesure où les auteurs de ces textes n'ont pour la plupart pas vécu les temps pré-chrétiens. Le seul qui pourrait les avoir vécus est l'auteur du manuscrit de Huahine et Borabora daté de 1846, ce qui suppose qu'il aurait appris à écrire dans les années 1820 et aurait eu suffisamment de familiarité avec l'écriture pour produire vingt ans plus tard le texte relativement élaboré que nous connaissons. Son manuscrit est pleinement le produit d'une culture de l'oralité dans le sens où les informations dont il disposait au sujet de la société traditionnelle lui sont parvenus oralement ; néanmoins, tant sur la forme que sur le fond, la rédaction proprement dite du manuscrit procède du processus d'apparition d'une culture chrétienne de l'écriture en langue tahitienne.

Analysant les rapports entre oralité et écriture au Moyen Âge européen, Paul Zumthor proposait de :

« distinguer trois types d'oralité, correspondant à trois situations de culture : l'un, primaire et immédiat, ne comporte aucun contact avec l'écriture [...] Il n'est pas douteux que la quasi-totalité de la poésie médiévale releva de deux autres types d'oralité, dont le trait commun est qu'ils coexistent, au sein du groupe social, avec l'écriture. Je les ai nommés respectivement oralité mixte quand l'influence de l'écrit y demeure externe, partielle et retardée, et oralité seconde quand elle se recompose à partir de l'écriture au sein d'un milieu où celle-ci tend à exténuer les valeurs de la voix dans l'usage et dans l'imaginaire. En inversant le point de vue, on dirait que l'oralité mixte procède de l'existence d'une culture "écrite" (au sens de "possédant une écriture"); l'oralité seconde, d'une culture "lettrée" (où toute expression est marquée plus ou moins par la présence de l'écrit). » (1987: 18-19)

Dans le cas polynésien envisagé ici, aucun texte autochtone ne relève exactement de l'oralité primaire et immédiate. Celle-ci ne pourrait concerner que les transcriptions de traditions (comme celles du Tahiti aux temps anciens de Teuira Henry, ou de notre ouvrage Huahine aux temps anciens) mais pas l'écriture par les autochtones dans leur langue.

L'oralité mixte est celle des traditions contées en tahitien par Tearapō dans les années 1960 ou de celles portées à l'écrit par Émile Hiro dans les années 1980 (et publiées en 1997) : le savoir de ces auteurs emprunte aussi à des écrits aisément identifiables (dans les deux cas, l'ouvrage de Teuira Henry ainsi que des articles du Bulletin de la Société des études océaniennes lus en français par ces auteurs ${ }^{34}$ ).

S'agissant des puta tupuna de Rurutu, les premiers d'entre eux, ceux rédigés à la fin du XIX ${ }^{\mathrm{e}}$ siècle, sont à inscrire au rang de l'oralité mixte, d'une culture née de la rencontre de la tradition orale polynésienne et de l'écriture biblique; en revanche, les manuscrits recopiés ou rédigés ultérieurement, sur la base de ces premiers puta tupuna, mériteraient d'être reconnus comme relevant de l'oralité seconde, puisque la voix provient principalement du texte.

Même lorsqu'ils passent par l'écrit, les auteurs de ces manuscrits ignorent les codes de référence livresque de la tradition scientifique occidentale. Ils continuent de se situer dans une logique où les savoirs relatifs à leur île, leurs ancêtres, n'ont pas de véritable auteur mais appartiennent au groupe dont ils font partie et dont ils se font le porte-voix momentané. Dès qu'on entre dans le domaine de la tradition orale, les auteurs polynésiens, qui travaillent pourtant dans l'intertextualité, ouvrent rarement les guillemets. Certains signent de leur nom personnel des textes de traditions dont ils ne sont pas les auteurs (d'ailleurs, ces œuvres collectives immémoriales, n'en ont pas, à proprement parler) mais plutôt les derniers relais : ainsi de Daniel Terooatea (1997) et Pare Walker (1997) pour Rurutu, qui tous deux apposent leur nom en bas de textes issus ou largement inspirés d'un puta tupuna d'un de leurs ancêtres, sans le mentionner, certainement parce qu'ils estiment que lui-même n'était qu'un dépositaire parmi d'autres de traditions qu'ils s'approprient à leur tour. Cet exemple nous conduit à une appréhension plus générale du savoir dans les sociétés de tradition orale ou encore partiellement porteuses de ce type de savoir.

Les sociétés modernes, occidentales, vivent aujourd'hui dans l'idée que le savoir n'appartient a priori à personne; il ne court donc pas le risque d'être confisqué ou monopolisé illégitimement. Dans ce type de pensée, tout savoir appartient potentiellement à celui qui se donne les

34. Ces auteurs traduisent parfois des éléments d'un texte initial (en français) comportant des informations si spécifiques ou des erreurs si manifestes (qu'ils reproduisent), que l'emprunt ne peut faire de doute. Dans le cas d'Émile Hiro, ses traditions des îles Sous-le-Vent empruntent aux articles de Marcantoni et Chesneau (1928). 
moyens d'y accéder par son travail, son courage, son talent. La connaissance est un domaine supérieur vers lequel tout individu peut se hisser, une richesse qui circule en quelque sorte en dehors des gens, au-dessus des gens, horizontalement. À l'inverse, dans les sociétés traditionnelles, le savoir, surtout lorsqu'il est relatif à l'origine des groupes sociaux, circule ou plutôt se diffuse de génération à génération. Il est constitutif de ces groupes et réciproquement ne saurait exister indépendamment d'eux. La perception des traditions y est verticale : ces savoirs sont certes immatériels, c'est-à-dire en quelque sorte situés au-dessus de la matérialité du groupe, mais ils se situent aussi dans la continuité verticale stricte de ces groupes, dont ils constituent la part spirituelle inaliénable. De là bien des débats, présents et à venir, en Océanie, sur les « véritables détenteurs » des « véritables traditions ».

\section{BIBLIOGRAPHIE}

BABADZAN Alain, 1979. De l'oral à l'écrit, les «puta tupuna » de Rurutu, Journal de la Société des Océanistes XXXv, 65, pp. 224-234.

_, 1982. Naissance d'une tradition. Changement culturel et syncrétisme religieux aux îles Australes (Polynésie française), Paris, orsTom, travaux et documents $154,313 \mathrm{p}$.

BARÉ Jean-François, 1987. Tahiti, les temps et les pouvoirs. Pour une anthropologie historique du Tahiti post-européen, Paris, ORSTOM, 543 p.

Chesneau Joseph (avec la participation de Pascal MarCantoni), 1928. Histoire de Huahine et autres îles Sous-le-Vent, Bulletin de la Société des études océaniennes 25, pp. 57-67, et 26, pp. 81-98.

Churchward James, 1969 (1 ère éd. 1926). Mu, le continent perdu, Paris, J'ai lu, collection L'aventure mystérieuse.

Collectif, 1997. Hei pua ri'i, Papeete, éditions Academie tahitienne - Fare vana'a, 224 p.

—, 1999. Dictionnaire Tahitien-Français, Papeete, éditions de l'Académie tahitienne, $574 \mathrm{p}$.

Devatine Flora, 1979. Problèmes rencontrés en Polynésie pour la conservation du patrimoine culturel et le développement des cultures océaniennes, Bulletin de la Société des études océaniennes 206, pp. 379-404.

Driessen Henk A. H., 1984. Comment percevoir l'autre ? Personnages nimbés de gloire et pirogues sans balancier. Prophéties d'avant les contacts dans les îles de la Société, Bulletin de la Société des études océaniennes 226, pp. 1481-1511, traduit de l'anglais par Robert Koenig (édition originale1982. Outriggerless Canoes and Glorious Beings. Pre-contact Prophecies in the Society Islands, Journal of the Polynesian Society XVII, 1, pp. 3-26).
EDDowes Mark, 1991. Marae Nuurua. Travaux partiels de restauration. Partial restoration of the marae complex, Papeete, Centre polynésien des sciences humaines Te Anavaharau, département Archéologie, $29 \mathrm{p}$.

Ellis William, 1972. À la recherche de la Polynésie d'autrefois, Paris, Publications de la Société des Océanistes 25, 2 vols., 943 p. (éd. originale 18291830. Polynesian Researches During a Residence of Nearly 8 Years in the Society and Sandwich Islands, Londres, Fisher \& Jackson, 4 vols.).

EMORY Kenneth, 1927. Traditional History of marae in the Society Islands, Honolulu, Bishop Museum, tapuscrit.

_, 1933. Stone remains in the Society Islands, Honolulu, Bishop Museum, Bulletin 116, 182 p.

_, 1938. The Tahitian Account of Creation by Mare, Journal of the Polynesian Society 47, 2, pp. 45-63.

- 1939. The Tuamotuan Creation Charts by Paiore, Journal of the Polynesian Society 48, 1, pp. 1-29.

_, 1940a. Tuamotuan Concepts of Creation, Journal of the Polynesian Society 49, 1, pp. 69-136.

-, 1940b. A newly discovered illustration of Tuamotuan Creation, Journal of the Polynesian Society 49, 4, pp. 569-578.

-, 1943. Additional illustrations of Tuamotuan Creation, Journal of the Polynesian Society 52, 1, pp. 1921

—, 1947. Tuamotuan Religious Structures and Ceremonies, Honolulu, Bishop Museum, Bulletin 191.

Goody Jack, 1994. Entre l'oralité et l'écriture, traduit de l'anglais par Denise Paulme, révisé par Pascal Ferroli, Paris, PUF, coll. Ethnologies, 323 p. (éd. originale 1993, The Interface between the Written and the Oral).

Henry Teuira, 1988 (1 ${ }^{\text {re }}$ éd. 1951). Tahiti aux temps anciens, Paris, Publications de la Société des Océanistes $1,722 \mathrm{p}$.

Hiro Émile Teriieroo, 1997a. E parau no Huahine, in Collectif, Hei pua ri'i, Académie tahitienne - Fare Vana'a, pp. 30-33.

_ , 1997b. Ra'iātea 'oia 'o Havai'i i muta'a ihora, in Collectif, Hei pua ri'i, Académie tahitienne - Fare Vana'a, pp. 105-119.

_ 1997. Huahine, 'o tei parau-ato'a-hia 'o Mata'irea, in Collectif, Hei pua ri'i, Académie tahitienne - Fare Vana'a, pp. 125-141.

GiLl Wyatt, 1911. E parau tupuna no to matou fenua, no Rurutu. Extracts of Dr. Wyatt Gill's papers (traduit en anglais par Teuira Henry), Journal of the Polynesian Society, pp. 136-139.

Laval Honoré, 1938. Mangareva. L'histoire ancienne d'un peuple polynésien. Mémoires ethnographiques, édités et annotés par le Dr Alfred Métraux, en collaboration avec le rév. père Maurice Desmedt, Braine-le-Comte - Paris, Maison des Pères des Sacrés-Cœurs - P. Geuthner, 378 p. 
McLuhan Marshall, 1977 (1 $1^{\text {re }}$ éd. 1967). La Galaxie Gutenberg, la genèse de l'homme typographique, 2 tomes, Paris, Gallimard, collection idées, 520 p. (éd. originale 1962, The Gutenberg Galaxy: The Making of Typographic Man, University of Toronto Press).

Manate Nootu, n.d. Manuscrit identifié comme Parau tupuna of the Manate Family, Rurutu, Peabody Museum, Salem (Massachusetts), Brunor Collection, 158 p., avec une introduction de Martin Brunor (1965).

Manutahi (ou Manu-Tahi) Charles Teriiteanuanua, 1982. Contes et légendes de la Polynésie. Le secret des livres tupuna. Te parau huna a te mau tupuna, préfacé par Claude Marere, Papeete, éd. Te hiroà mā'ohi tumu, $173 \mathrm{p}$.

_, 1992. Te parau huna o te ao mä'ohi. Le mystère de l'univers mā'ohi, Papeete, éd. Te tumu o te hiroà mā’ohi - Veiā Ra'i, 113 p.

_, 1997. Te parau o Papenoo, e peho no Tahiti. L'histoire de la vallée profonde de Papenoo, île de Tahiti, Papeete, éd. Te tumu o te hiroà mā'ohi, $142 \mathrm{p}$.

—, 2005. Te parau o te mau vähi faufaa no te mau tupuna i Moorea. L'histoire des sites et des ancêtres de l'île de Moorea, Papeete, éd. Te tumu o te hiroà mā'ohi, 158 p.

Marau TaAroa (Salmon), 1971. Mémoires de Marau Taaroa, dernière reine de Tahiti, Paris, Société des Océanistes 27, 294 p.

Mauer Daniel, 1982. Aimer Tahiti. Tahiti les yeux ouvert, Paris, Nouvelles éditions latines, $253 \mathrm{p}$.

Montillier Pierre, 1999. Te reo tahiti 'àpī. Dictionnaire $d u$ tahitien nouveau et biblique, Papeete, à compte d'auteur, $365 \mathrm{p}$.

Nicole Jacques, 1988. Au pied de l'écriture. Histoire de la traduction de la Bible en tahitien, Papeete, Haere po no Tahiti, 338 p.

OLIVER Douglas, 1974. Ancient Tahitian Society, 3 vols., Honolulu, The University Press of Hawaii, 1419 p.

ONG Walter J. 1988 ( $1^{\text {st }}$ ed. 1982). Orality and Literacy: The Technologizing of the Word, New York, Methuen.

O'ReILly Patrick et Raoul TeIssIER, 1962. Tahitiens. Répertoire bio-bibliographique de la Polynésie française, Paris, Musée de l'Homme, Société des Océanistes, $534 \mathrm{p}$.

Parsonson G.S., 1967. The literate revolution, Journal of Pacific History 2, pp. 39-57.

PoEtai Teriimana, n.d. (vers 1965). Manuscrit identifié comme Teriimana's book. History. Vanilla business, Peabody Museum, Salem (Massachusetts), Brunor Collection, reel \# 3. 122, 175 p.

Ratinassamy Martine et Hiriata Millaud, 2001. Porapora i te fānau tahi. Mille ans de mémoire, texte de Martine Ratinassamy, traduction et normalisations graphiques de Hiriata Millaud, Papeete, Cahier du Patrimoine 2, ministère de la Culture et de l'Enseignement supérieur de la Polynésie française, $78 \mathrm{p}$

Richaud-TuHeIAVA Vahi Sylvia, 1999. Essai d'analyse de la parole, Parau, dans l'univers de l'île de Maupiti en Polynésie française. Un premier champ de construction ethno-linguistique. mémoire de DEA, université française du Pacifique, $120 \mathrm{p}$.

SAURA Bruno et al., 2000. Histoire et traditions de Huahine et Porapora, traduction de Patrick Daubard, normalisations graphiques de Hiriata Millaud, préface et édition critique de Bruno Saura, Papeete, Cahier du Patrimoine 1, ministère de la Culture et de l'Enseignement supérieur de la Polynésie française, $89 \mathrm{p}$.

_, 2003. La dynastie des Tama-toa de Ra'iātea (îles Sous-le-Vent). Puta 'à'amu nō te 'ōpū ari'i Tama-toa nō Ra'iātea, Préface, édition critique et traduction de Bruno Saura, normalisations graphiques de Hiriata Millaud, Papeete, Cahier du Patrimoine 6, ministère de la Culture et de l'Enseignement supérieur de la Polynésie française, 229 p.

SAURA Bruno, 1995. Les règles coutumières en Polynésie française, in Paul de Deckker (éd.), Coutume autochtone et évolution du droit dans le Pacifique Sud, Paris, L'Harmattan, pp. 95-131.

_, 1998. The Emergence of an Ethnic Millenarian Thinking, and the Development of Nationalism in Tahiti, Pacific Studies 21, 4, pp. 33-66.

—, 2002. Continuity of Bodies: the Infant's Placenta and the Island's Navel in Eastern Polynesia, Journal of the Polynesian Society 111, 2, pp. 127-145.

_, 2004. La société tahitienne au miroir d'Israël. Un peuple en métaphore, Paris, CNRs éditions, coll. Ethnologie, $302 \mathrm{p}$.

—, 2005a. Des Incas en Polynésie ? Spéculations occidentales et traditions autochtones de Rurutu d' « hommes rouges » venus de l'Est, in $\mathrm{P}$. Vérin et $\mathrm{R}$. Veccella (éds), L'Amérique hispanique et le Pacifique. Hommage à Hugo Neira, Paris, Karthala, pp. $45-68$

-, 2005b. Huahine aux temps anciens, Papeete, Cahier du Patrimoine 8, service de la Culture et du Patrimoine de la Polynésie française, 287 p.

_, 2008 (à paraître). Les généalogies de Rurutu sontelles vraies ? Ou comment on écrit l'histoire en Polynésie, Bulletin de la Société des études océaniennes, $19 \mathrm{p}$.

Stimson John Francis, 1933a. The cult of Kiho Tumu, Honolulu, Bishop Museum, Bulletin 111, 63 p. (Version annotée par Emory, Bishop Museum Archives, Fonds Stimson Ms. Sc. Box 1.1)

_, 1933b. Tuamotuan Religion, Honolulu, Bishop Museum, Bulletin 103.

Tearapō (Teauna Pouira, dit Tearapō), 1997. Papa'oā, Ha'apape, Papeete, Centre polynésien des sciences humaines - Te Anavaharau, $50 \mathrm{p}$.

-, 1999. Parau tumu nō Teaharoa, Papeete, Centre polynésien des sciences humaines - Te Anavaharau, $60 \mathrm{p}$. 
—, 2000a. Parau tumu nō Tahiti iti, Papeete, Centre polynésien des sciences humaines - Te Anavaharau, $42 \mathrm{p}$.

—, 2000b. Parau tumu nō Tãfa'i, Papeete, Centre polynésien des sciences humaines - Te Anavaharau, $34 \mathrm{p}$.

_, 2000c. Parau nō te mau terera'a nā te moana, Papeete, Centre polynésien des sciences humaines Te Anavaharau, $42 \mathrm{p}$.

_, 2000d. Te parau nō te mau ta'amotu, Papeete, Centre polynésien des sciences humaines - Te Anavaha$\mathrm{rau}, 55 \mathrm{p}$.

Teroontea Daniel, n.d. (vers 1997). Te va'a ta'ata mātāmua $i$ tapae $i$ Rurutu, Papeete, à compte d'auteur, $191 \mathrm{p}$.
VÉRIN Pierre, 1969. L'ancienne civilisation de Rurutu (îles Australes - Polynésie française). La période classique, Paris, ORSTOM, 318 p.

Walker Ta'aria (dite Pare), 1997. 'ÁA'ai nō te mau ta'ata mātāmua i tae i Rurutu, in Collectif, Hei pua ri'i, Papeete, Académie tahitienne - Fare Vana'a, pp. 91-95.

_, 1999. Rurutu, mémoires d'avenir d'une île Australe, Papeete, Haere Po, 158 p.

Zumthor Paul, 1987. La lettre et la voix. De la "littérature " médiévale, Paris, Éditions du Seuil, $346 \mathrm{p}$. 
